\title{
The Cognitive Phenotype of Down Syndrome: Insights from Intracellular Network Analysis
}

\author{
Avi Ma'ayan,* Katheleen Gardiner, ${ }^{\dagger}$ and Ravi Iyengar* \\ *Department of Pharmacology and Biological Chemistry, Mount Sinai School of Medicine, New York, New York 10029; and \\ ${ }^{\dagger}$ Eleanor Roosevelt Institute at the University of Denver, University of Colorado at Denver and the Health Sciences Center, \\ Denver, Colorado 80206
}

\begin{abstract}
Summary: Down syndrome (DS) is caused by trisomy of chromosome 21. All individuals with DS exhibit some level of cognitive dysfunction. It is generally accepted that these abnormalities are a result of the upregulation of genes encoded by chromosome 21. Many chromosome 21 proteins are known or predicted to function in critical neurological processes, but typically they function as modulators of these processes, not as key regulators. Thus, upregulation in DS is expected to cause only modest perturbations of normal processes. Systematic approaches such as intracellular network construction and analysis have not been generally applied in
\end{abstract}

DS research. Networks can be assembled from highthroughput experiments or by text-mining of experimental literature. We survey some new developments in constructing such networks, focusing on newly developed network analysis methodologies. We propose how these methods could be integrated with creation and manipulation of mouse models of DS to advance our understanding of the perturbed cell signaling pathways in DS. This understanding could lead to potential therapeutics. Key Words: Down syndrome, systems biology, graph theory, text-mining, Bayesian networks, qualitative modeling.

\section{INTRODUCTION}

Down syndrome (DS) is caused by an extra copy of all or part of the long arm of human chromosome 21. With an incidence of approximately 1 per 800 live births, DS is the most common neurodevelopmental disorder. ${ }^{1,2}$ Structurally, while the brain is largely normal at birth, subsequent failures in postnatal development result in reduced volumes of the hippocampus, cerebellum, and prefrontal cortex; reduced neuronal densities in specific regions that include the hippocampus, cerebellum, and basal forebrain; and reduced dendritic branching, length, and spine densities in the hippocampus (reviewed in Nadel $^{3}$ and Benavides-Piccione and coworkers ${ }^{4}$ ). All individuals with DS exhibit some level of cognitive dysfunction, although there is considerable variability in the severity. The average IQ is approximately 50 , but ranges over 40 points from severely impaired to low normal intelligence. ${ }^{5,6}$ Cognitive dysfunction is manifested as deficits in specific tasks requiring a functional hippocam-

Address correspondence and reprint requests to: Ravi Iyengar, Ph.D., Department of Pharmacology and Biological Chemistry, Mount Sinai School of Medicine, New York, NY 10029. E-mail: ravi.iyengar@mssm.edu pus, specific strengths and weaknesses in language skills, implicating regions of the prefrontal and temporal cortices and the cerebellum, and additional deficits suggesting impaired prefrontal cortex function. ${ }^{7-9}$ Cognitive dysfunction in DS thus presents a specific constellation of features that distinguish it from other intellectual disabilities, such as Williams syndrome and Fragile X. Also common to all individuals with DS is the development of the neuropathology of Alzheimer's disease (AD) by the age of 30-40 years, although only 50\% also develop an AD-like dementia (reviewed in Lott and Head ${ }^{10}$ ). Other abnormalities include loss of cholinergic markers in the basal forebrain, a higher frequency of delayed myelination, and increased incidences of seizures and autism. ${ }^{3,11}$

The working hypothesis in most DS research is that expression of chromosome 21 genes is increased approximately $50 \%$ due to gene dosage but that overexpression of only a subset of trisomic genes is responsible for the phenotypic features that characterize DS. In the most recent published report, almost 400 genes were annotated within 21 q but only $\sim 170$ were protein coding and conserved in the orthologous regions of the mouse genome. ${ }^{12}$ While total gene numbers may increase, new additions are likely to encode functional RNAs or am- 
TABLE 1. Chromosome 21 Genes as Modulators

\begin{tabular}{ll}
\hline Pathway/Process & \multicolumn{1}{c}{ Chromosome 21 Proteins } \\
\hline MAP kinase & ITSN1, TIAM1, DYRK1A \\
Calcineurin activity & DSCR1, PCP4, SOD1, APP \\
Calcium signaling & S100B, TRPC7, C21orf25 \\
Calmodulin activity & PCP4 \\
Glutamate receptor & GRIK1 \\
Axon guidance & DSCAM \\
Dendritic spines & TIAM1, DYRK1A, APP \\
Adult neurogenesis & OLIG1,2 \\
NMDA receptors & TIAM1, Calcineurin signaling \\
Dopamine receptors & CLIC6 \\
Seizures & S100B, CSTB, GIRK2 \\
Potassium channels & KCNJ6, KCNE1,2 \\
Mitochondrial function & GABPA, DSCR1, ATP5J, ATP50, NDUVF3, CRYZL, MRPS6, L39 \\
BDNF & Calcineurin signaling, MAPK signaling, ER \\
CREB & DYRK1A, DSCR1, MAPK \\
Elk, ER, GR & SUMO3, DYRK1A, NRIP1, MAPK signaling, Calcineurin signaling \\
\end{tabular}

biguous open reading frames and/or not to be conserved in mouse. Current approaches to gene-phenotype correlations focus on conserved protein-coding genes because these can be modeled in mouse with well-established technologies and reagents. In considering candidates among the protein-coding genes, two points are important. First, analysis of DS due to partial trisomy $21 \mathrm{q}$ might be informative. However, cases are rare and rigorous correlation of trisomic gene content with specific phenotypic features has not been carried out, in particular for cognitive deficits. ${ }^{13-15}$ Indeed, no significant segment of $21 \mathrm{q}$ can be discounted from possibly containing relevant genes. ${ }^{16}$ Second, results of large-scale expression experiments comparing trisomic human and mouse tissues to controls generally support the hypothesis of increased mRNA levels due to gene dosage, ${ }^{17-22}$ although there are gene- and tissue-specific exceptions. An additional consideration, however, is that there are significant variations in expression levels of chromosome 21 genes even among normal controls. ${ }^{23}$ Gene dosage effects at the protein level have not been examined comprehensively but have been verified for individual genes, although, again, there are gene- and tissue-specific exceptions (for example, see O'Leary and coworkers ${ }^{24}$ ).

The 170 protein-coding genes on chromosome 21 function in numerous protein complexes, biochemical pathways, and cellular processes. A subset of these will be relevant to neurodevelopment and synaptic plasticity. While knowledge is far from complete, some functional information exists for the majority of the protein-coding genes. In vitro studies with human chromosome 21 genes, or mouse orthologs, in vivo studies with orthologs in model organisms, and bioinformatics approaches have implicated chromosome 21 proteins in MAP kinase pathways, calcium and calcineurin signaling, neurogenesis, axon guidance, development of dendritic spines, NMDA and dopamine receptor functions, potassium channels, and mitochondrial function, as well as in regulation of learning and memory genes such as BDNF, CREB, Elk, and the estrogen and glucocorticoid receptors (Table 1) (also see Gardiner and Costa, ${ }^{25}$ Nikolaienko and cowork$\mathrm{ers}^{26}$ and Gardiner and coworkers ${ }^{27}$ ). It is of particular interest to note, however, that chromosome 21 proteins are not central players. They impact the processes peripherally, often as one of several proteins with similar roles or as one of several subunits in a complex. For example, the chromosome 21 encoded DSCR 1 is a modulator of calcineurin activity. Other, nonchromosome 21 genes, DSCR1L1, ZAKI-4, and CAIN/Cabin, also have been identified as inhibitors of calcineurin activity. ${ }^{28}$ The Drosophila ortholog of c21orf2 was recently shown to be a negative regulator of $\mathrm{Wnt}$, one of several identified. ${ }^{29}$ The chromosome 21-encoded ionotropic glutamate receptor subunit, GRIK1, is one of five kainate responsive subunits that form heteromeric complexes. ${ }^{30}$ Thus, chromosome 21 proteins are modulators, not directors, of critical neurological processes and their increased expression perturbs pathways; it does not obliterate them (FIG. 1). A second important point is that many chromosome 21 proteins mutually interact and/or impact the same pathway(s) or share the same targets (FIG. 1). Thus, representation of gene-phenotype correlations in DS requires a systems approach and should have as a goal gene-pathway/network-phenotype correlations.

Because of the large number of candidate genes and the complexity of the cognitive dysfunction, DS represents a significant challenge for systems biology. Integration of knowledge about protein interactions and the hypotheses produced by computational modeling regarding pathway perturbation can be tested in mouse models. These mouse models can be further manipulated by genetic and pharmacological means to verify candidate 


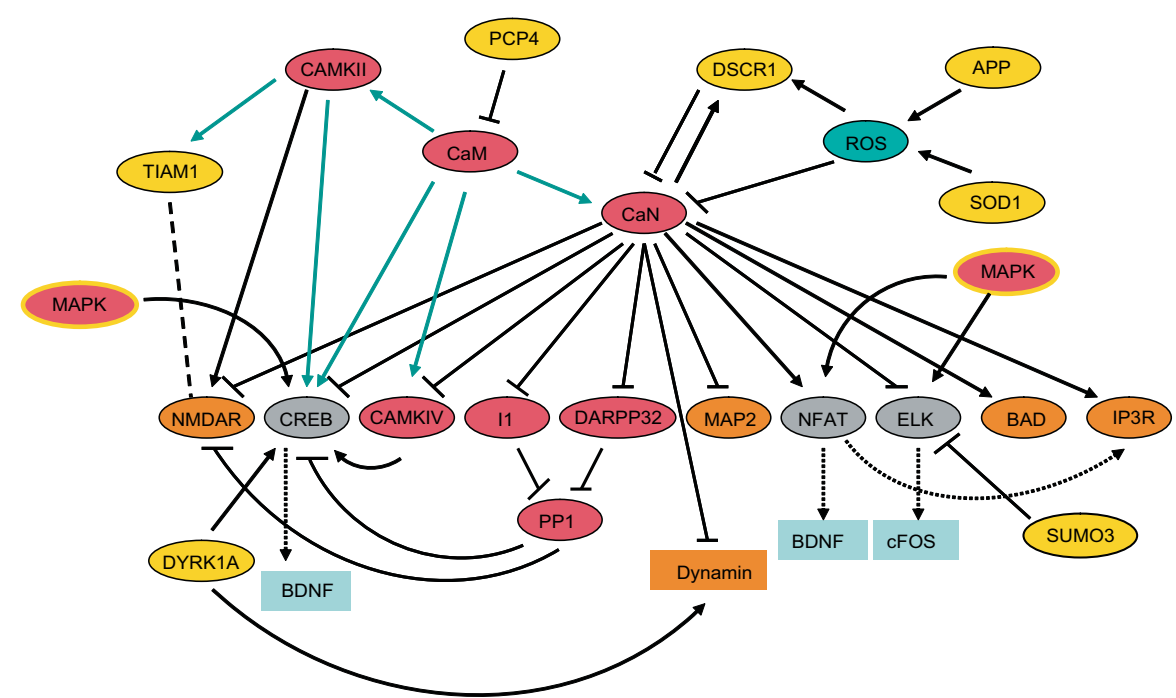

FIG. 1. Chromosome 21 genes, depicted in yellow, act as modulators of key signaling pathways and impact common targets involved in neuronal synaptic plasticity.

pathway perturbations and test for their amelioration. Can we understand how proportions of interacting components leading to functional networks are altered in DS to affect phenotypic behavior? The answer to this question is a cautious "yes." There is increasing evidence from studies of the most complex DS mouse model (Ts65Dn, see below) that molecular and cellular abnormalities, whatever their origins in the developmental program, can be potentially corrected. Abnormalities include impaired adult neurogenesis, loss of functional markers of the cholinergic neurons, decreased cell numbers in the cerebellum, and possibly even spatial learning. ${ }^{31-34}$ Treatment with fluoxetine, NGF, an agonist of sonic hedgehog, and estrogen, respectively, were shown to provide improvements. A systematic biological approach will define pathway perturbations and identify new, more effective targets for development of therapeutics. It is reasonable to expect that some of these may at least ameliorate, if not reverse, the consequences of abnormalities in gene and protein levels in DS.

\section{FROM GENES TO PATHWAYS AND NETWORKS}

A common obstacle in understanding how changes at the gene level are manifested as altered phenotypes is that the observed phenotype often does not directly involve the function of the "disease" gene. Here, systematic approaches can be quite useful in identifying distal connections and defining how seemingly unrelated cellular components may actually be linked through their participation in cellular networks. This is likely to be particularly relevant for DS because many of the gene products of chromosome 21 are modulators of cell signaling pathways. To develop this line of thinking it is necessary to integrate several computational disciplines. These include bioinformatics, graph theory analyses of networks, quantitative differential equation-based models, and statistical models using Bayesian logic. These theoretical approaches in turn need to be integrated with multiple biochemical and molecular biological highthroughput and gene-specific approaches focused on detailed characterization of biomolecular interactions. In this review we describe the current approaches for development and analyses of functional biochemical networks and their potential application to DS research.

\section{DATABASES/NETWORKS OF MAMMALIAN PROTEIN-PROTEIN AND LIGAND-PROTEIN INTERACTIONS}

Large-scale mammalian in silico cellular network connection maps describing protein-protein and ligan$\mathrm{d}$-protein interactions are emerging both from information in legacy biomedical research articles, describing only few interactions and proteins, and from highthroughput wet lab methods capable of identifying hundreds to thousands of interactions in a single experimental setting. High-throughput experimental methods include, for example, construction of networks from Affymetrix microarray data, ${ }^{35-36}$ highthroughput yeast-2-hybrid screens, as applied to yeast, fly, and worm, ${ }^{37-39}$ and recently to human cells, ${ }^{40,41}$ immobilized metal affinity chromatography followed by liquid chromatography-mass spectrometry to identify phosphorylated peptides, ${ }^{42}$ multiparameter flow cytometry, ${ }^{43}$ and proteome chip to identify phosphorylation sites. ${ }^{44}$ The high-throughput methods can identify binary interactions. The datasets produced are most commonly qualitative. Recently, Jones and co- 
workers ${ }^{45}$ were able to measure, in high-throughput, the binding rate constants of ERB receptors to their substrates generating a quantitative dataset. Literature text-mining methods using the development of artificial intelligence based tools are used to automatically extract interactions from PubMed abstracts and fulltext research papers. ${ }^{46-49}$ Alternatively, text-mining of interactions from experimental literature can be extracted manually. ${ }^{50-52}$ The automatic methods, compared with the manual methods, contain higher levels of false positives and lower levels of false negatives. ${ }^{53}$ False positives are interactions that are not relevant in the biological context but exist in the data. False negatives are missed interactions that exist in the literature.

Mammalian protein-protein interaction networks are quickly growing in size. Most of these databases are publicly available for download and analysis. Proteinprotein and/or ligand-protein interactions networks are stored in several templates. The Systems Biology Markup Language (SBML) ${ }^{54}$ is an Extensible Markup Language (XML) (http://www.w3.org/XML) standard used by biochemical modeling software packages (http:// www.sbml.org) to store biochemical interactions. SBML provides direct conversion of biochemical interactions to be quantitatively simulated. The Systems Biology (SB) toolbox was developed for modeling SBML XML files. ${ }^{54}$ The Database of Quantitative Cellular Signaling, DOQCS, is a relational database management system (RDBMS) that stores molecular regulatory interactions in a format also readily available for quantitative simulations. ${ }^{55}$ SBML and DOQCS models rely on detailed representation of biochemical reactions that include rate constants and concentrations. Alternatively, more simplified schemas that only represent the binary relationships between interacting components have been developed. Some leading databases that follow this approach are $\mathrm{BIND},{ }^{56} \mathrm{HPRD}^{50}{ }^{5} \mathrm{MINT},{ }^{57}$ IntAct, ${ }^{58} \mathrm{DIP},{ }^{59,60}$ KEGG, ${ }^{61}$ and PPID. ${ }^{62}$ Most of these databases describe mainly binding interactions and yield undirected graphs, wherein the direction of the flow of information is not specified. Records in these databases typically include two interacting molecular components, their accession codes, and the PubMed ID of the article that describes the interaction. The DIP database, for example, implemented an XML schema called XIN, which follows the more simplified representation of interactions data. ${ }^{59} \mathrm{Re}-$ cently, HUPO (Human Proteome Organization) developed another XML standard called PSI-MI (Proteomics Standards Initiative-Molecular Interactions) for storing protein interactions. This standard compromises between SBML and XIN and is adopted already by several databases and labs. ${ }^{63}$ Science magazine's Signal Transduction Knowledge Environment (STKE) is a web-based journal that publishes cell signaling connection maps.
The data presented as maps on STKE are stored in a relational database called CMADES (connection maps authority data entry system). The schema to store the data follows the more simplified nodes and links paradigm. ${ }^{51}$ STKE data are exportable to SBML compatible XML format. Bader and coworkers ${ }^{64}$ collected and maintain a list of databases of protein-protein and ligand -protein interactions of mammalian and other organisms.

The richness of these emerging datasets could be a starting point to tackle the effects that upregulation of chromosome 21 genes in DS have on the entire human protein-protein and ligand-protein interactions network. Figure 2 shows a protein-protein and ligand-protein interaction network map made of interactions from three databases: a database of mammalian cell-signaling interactions in CA1 neurons we developed manually, ${ }^{52} \mathrm{BIND}$ human protein-protein interactions dataset, ${ }^{55}$ human protein reference database HPRD, ${ }^{50}$ and PPID. ${ }^{62}$ The chromosome 21 genes that appear in those databases were pulled out of the network map and are highlighted in orange. APP and the IFN alpha receptor are the most connected chromosome 21 genes. This is probably due to research interest in $\mathrm{AD}$ where amyloid plaques and neurofibrillary tangles observed in postmortem $\mathrm{AD}$ patients contain peptide chains from the APP gene product protein precursor. Amyloid plaques were observed also in postmortem DS individuals. ${ }^{10}$ Down syndrome is also implicated with immunodeficiency. IFNAR1 effects are also well studied due to their key involvement in triggering immune response where the gene is expressed in most immune cells. Gerdes and coworkers ${ }^{65}$ demonstrated increase IFN alpha and beta signaling in DS lymphocytes. Zooming into network maps such as the map drawn in Figure 2 to understand specific modules in the network that may be affected in DS is the next step in understanding how gene dysregulation leads to cellular changes that result in phenotypes observed in DS.

\section{NETWORK ANALYSIS USING GRAPH THEORY}

Since the late 1990s, the approach of abstracting complex systems to networks, resulting in directed or undirected graphs made of nodes and links, is increasingly employed to analyze systems from a range of scientific fields. These applications nicely fit the molecules to nodes and interactions to links simplification used to describe intracellular mammalian interactions networks. ${ }^{66}$ Watts and Strogatz ${ }^{67}$ used two previously defined global statistical properties of graphs to characterize networks: clustering coefficient and characteristic path length. It was found that biological interaction networks have higher clustering coefficients and similar characteristic path lengths expected if the networks would be randomly rewired. An initial striking result 


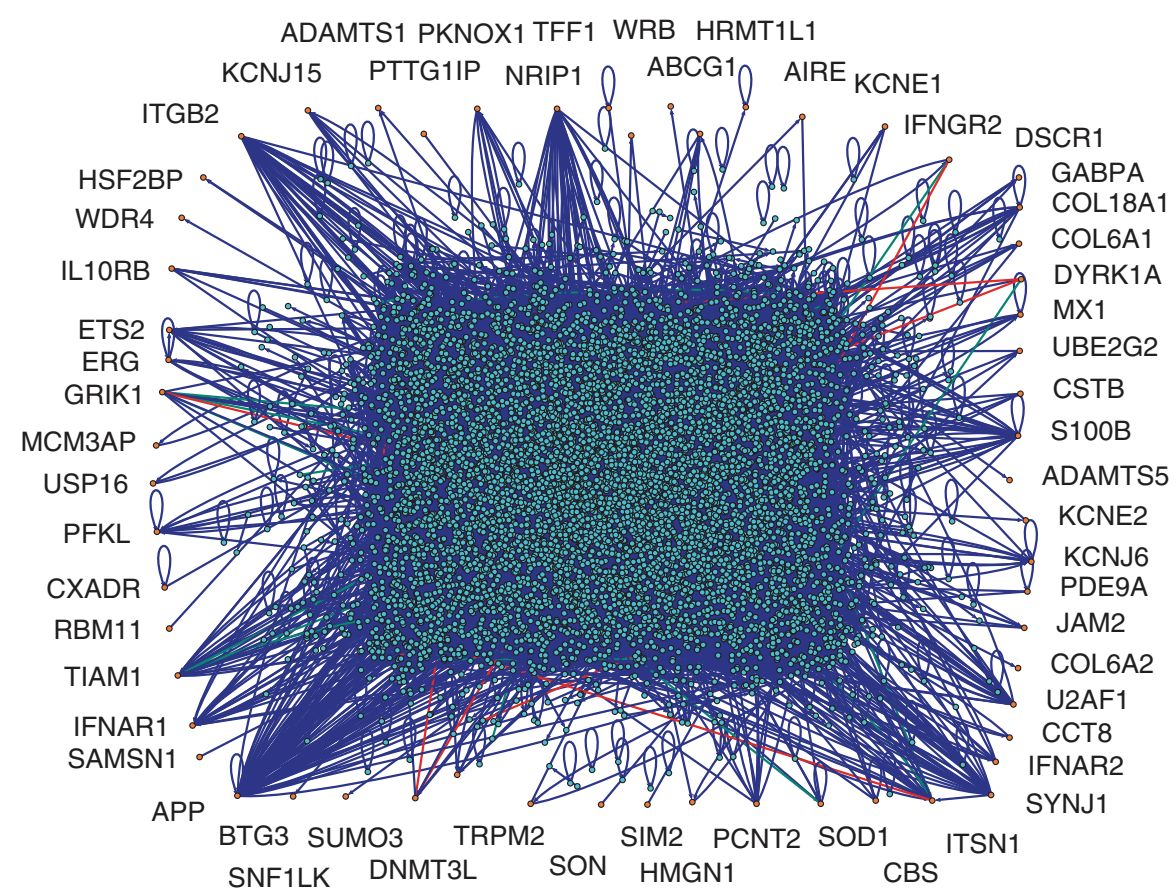

FIG. 2. Fifty-nine chromosome 21 genes have annotated interactions in a network created from HPRD, ${ }^{50}$ BIND, ${ }^{56}$ PPID, ${ }^{62}$ and a CA1 mammalian neuronal cell signaling network. ${ }^{52}$ The network contains 8260 nodes, representing mammalian proteins and small molecules such as ligands and second messengers (i.e., cAMP, $\mathrm{Ca}^{2+}$ ), and 34,706 direct molecular interactions. Blue arrows represent undirected interactions, while red (inhibition) and green (activation) represent direct interactions. A web-based interface that allows users to navigate and explore more details about these interactions is available on http://amp.pharm.mssm.edu/ds. A more comprehensive resource for chromosome 21 proteins and their primary and secondary interactions is available at http://chr21db.cudenver.edu. This web resource includes also relevant orthologs interactions from nonmammalian model organisms.

from the analysis of biochemical interaction networks is that network nodal connectivity distribution fits a powerlaw. ${ }^{68}$ Such networks are termed scale-free. Another approach for analysis of complex systems abstracted to network maps is characterization of motifs. Network motifs are subsets of interactions involving several different components. Alon's group ${ }^{69}$ was the first to propose this approach for analyzing biochemical interactions networks. They initially analyzed a gene regulatory network of bacteria. It is also possible to break up large-size biochemical interaction maps into subnetworks based on specific criteria such as limiting the number of steps from a receptor to a transcription factor and then searching for network motifs only in those subnetworks. ${ }^{52}$ Major attention is targeted toward the hubs: the highly connected nodes in protein-protein, ligand-protein, and gene regulatory networks. Han and coworkers ${ }^{70}$ distinguished between party hubs and date hubs. Party hub proteins interact with many other proteins in the same compartment and at the same time, whereas date hubs interact with many other proteins at different times and places in the cell. When network maps include directionality of the links it is possible to separate hubs based on their in-links and out-links. Borneman and coworkers ${ }^{71}$ found that hubs with many in-links are often master regulators, such as the transcription factors MyoD or NeuroD. By looking at the participation of chromosome 21 proteins in net- work motifs, in specific subnetworks, and whether chromosome 21 genes are date or party hubs, as well as their relations with master regulators, it may be possible to understand how upregulation of chromosome 21 genes affects the dynamics of signaling and gene regulatory programs. The use of graph theory approaches to identify regulatory motifs may be a good starting point for understanding how chromosome 21 genes regulate neuronal processes.

\section{QUALITATIVE AND QUANTITATIVE MODELING}

Developing dynamic models of mammalian cells is an ultimate goal of systems biology. ${ }^{72}$ If such models can correctly capture the relationships of many components in a cell, it may be possible to track where the trajectory of trisomy 21 cells diverges from the normal cells. Simulation and modeling of biochemical networks as dynamic systems range from very simple qualitative modeling approaches (i.e., Boolean networks) to extremely complex formalisms (i.e., 3D stochastic reaction schemes). ${ }^{73}$ Qualitative simulations involve construction of models based on a defined set of constraints and typically are applied to network components in directed graphs. One straightforward approach is to convert directed graphs to coupled lin- 
ear differential equations. ${ }^{74}$ Qualitative simulations do not require all of the exact parameters, such as initial concentrations of components and rate constants of reactions. An example is QSIM, developed by Kuipers. ${ }^{75}$ In contrast to standard models based on ordinary differential equations, traditionally used in quantitative simulation of biochemical reactions, QSIM uses qualitative differential equations (QDEs). These are variable representation of ODEs. QSIM is an algorithm developed under the umbrella of a subfield of artificial intelligence called qualitative reasoning (QR). The idea behind QR is that the human mind uses qualitative reasoning to function in a quantitative environment, mimicking the "common-sense" we use as humans to qualitatively reason about quantitative phenomena in our environment and applying these concepts to algorithm development. QSIM was implemented to analyze systems in a variety of fields, including electrical engineering and physics. King and coworkers $^{76}$ implemented the QSIM algorithm to model glycolysis. Another qualitative modeling approach to network modeling and simulation is Boolean networks. Albert and Othmer ${ }^{77}$ showed that Boolean network modeling could reasonably reproduce the same network behavior that more traditional ODE simulations uncovered. They implemented a Boolean network for the cascade pattern of segment polarity genes in Drosophila melanogaster. The key in their implementation was to setup the correct Boolean functions that connect variables. Grefenstette and cowork$\mathrm{ers}^{78}$ implemented Boolean network models that include dimerization of gene products and binding of different dimers to promoter sequences. Implementing Boolean network models with increasingly more realistic properties of the underlining biology can make these models more accurate and relevant. Another type of qualitative/semiqualitative simulation method is using Petri-nets. Petri-nets are networks containing places (nodes) that exchange tokens based on rules (functions) that connect the places. The network places are graphically represented as directed graphs. Oliveira and coworkers ${ }^{79}$ used Petri-nets to model parts of the EGFR-MAPK quantitative model developed by Bhalla and Iyengar. ${ }^{80}$ Oliveira et al. ${ }^{79}$ did not require kinetic rates, weights for the connections, or probabilities for passing tokens from one place to another. The authors were able to identify the critical network "pinch-points," which are EGFR internalization and SOS production. Goss and Peccoud ${ }^{81}$ used Stochastic Petri-nets (SPNs) to simulate biochemical systems that typically are analyzed by ODEs. Stochastic Petri-nets are an extension to Petri-nets in which tokens are moving from place to place stochastically. In their implementations, transition functions had weights. Such qualitative modeling methods may be useful for identifying targets that may be suitable for modulation of multiple regulatory motifs, such as those found in networks that induce disease state.

Quantitative models of large systems are considerably harder to develop because they require large sets of relatively precise data that have yet to be obtained. The required experimental data fall into several categories. These include concentrations of cellular components, the rates of their interactions, including rates of enzyme and binding activities, their locations within cells, and rates of regulated movement between cellular compartments. Despite these limitations, the number of quantitative differential equation-based models has been growing steadily, and high-throughput experiments that measure rate constants are becoming a reality. ${ }^{45}$ Such models will be essential for drug development from network models.

\section{MODELING SIGNALING NETWORKS WITH BAYESIAN NETWORKS ANALYSIS}

Holland $^{82}$ described how cell signaling networks typically modeled by computer simulations could also be modeled by classifier systems. Classifier systems share many aspects of cell signaling networks such as parallelism and coordination, conditional actions, modularity, and adaptation. Exploratory statistical models built using these concepts can provide insight into the operation of perturbed pathways in DS. One of the most commonly used classification methods for this purpose is Bayesian networks (BN). Construction of $\mathrm{BN}$ from experimental measurements of mRNA levels, as a time-series or under different perturbations, to reverse-engineer gene regulatory networks from microarray data are the most common approach so far to rebuild networks from these data. ${ }^{83-85}$ Bayesian networks are acyclic graphs in which nodes represent the experimentally measured variables and links are probabilistic influences of variables on each other. Woolf and coworkers ${ }^{86}$ used this approach to build a BN from a multivariant dataset of 28 signaling proteins under 16 combinations of experimental conditions applied to mouse embryonic stem cells. ${ }^{87}$ These cells can be driven to self-renewal or to differentiation in culture based on the extracellular media provided (i.e., stimulation by extracellular ligands). The authors searched for network topology and probabilities to connect variables to best fit the experimental results. The resulting network was validated against shuffled networks and helped the authors to hypothesize about the outcome of differentiation versus self-renewal under conditions not yet tested experimentally. Sachs and coworkers ${ }^{88}$ used the BN approach to study the relationships between proteins and phospholipids, and the directionality of their links, after T-cell activation of naïve T-cells. Sachs and coworkers ${ }^{88}$ used data from single cell measurements using flow cy- 
tometry to measure the phosphorylation levels of key signaling nodes (proteins and phospholipids). ${ }^{43}$ The authors then determined hierarchical ordering of signaling components by applying experimental perturbations, such as knocking out a protein, by either pharmacological agents or RNA interference. They were able to determine which proteins are upstream or downstream in the signaling network using statistical correlations. Dynamic Bayesian networks $(\mathrm{DBN})^{89}$ attempt to solve some limitations of standard BN analysis. Dynamic Bayesian networks analysis is applied to multivariant time-series data. The idea is to identify correlations between variables at different time points. For example, if variable $x$ is up at time point $t 1$ and variable $y$ is up at time point $t 2$, it is possible that variable $x$ upregulates variable $y$. Dynamic Bayesian networks was implemented by Zou and Conzen ${ }^{90}$ to infer a gene regulatory network function from the yeast cell cycle dataset. The first implementation of DBN in biology is attributed to Murphy and Mian. ${ }^{91}$ Bayesian networks do not perform well when only few time points are available, while thousands of variables are measured (i.e., time-series microarrays data). Segal and coworkers ${ }^{92}$ recently suggested a potential solution. Instead of treating each variable independently, variables are grouped into modules such that the BN is constructed to connect the modules. Bayesian networks application to experimental results is essentially statistical. Another elaborate statistical method was used by Janes and coworkers ${ }^{93}$ to study cell signaling axes of apoptosis. The author analyzed 7980 experimental measurements by constructing high-dimensional vectors from the data to understand the trajectory of cellular response to different stimuli that induce either apoptosis or promote cell survival. Such approaches may be useful in identifying where cellular dynamics trajectories are deformed in DS.

\section{COMBINING QUALITATIVE NETWORK MODELING WITH EXPERIMENTS FROM LEGACY LITERATURE}

Using a combination of high-throughput experimental results with literature-based networks extracted from single gene studies that describe interactions between only a few proteins is expected to produce the most potent computational models. ${ }^{94}$ This approach to constructing and validating models is also referred to as reverse engineering. Kurata and coworkers, ${ }^{95}$ D'haeseleer and coworkers, ${ }^{96}$ and others distinguish between forward engineering of biochemical networks and reverse engineering of biochemical networks. Forward engineering is when detailed models are constructed with the kinetic rates and concentration of the molecular species are assembled in silico from experiments. These models are constructed to analyze potential behavior of a relatively small set of interacting proteins. Reverse engineering, on the other hand, involves the construction of larger-scale models that are simulated without kinetic parameters. Gat-Viks and coworkers ${ }^{97}$ applied this approach to study lysine biosynthesis in yeast. They used low-throughput extensive literature searches to build an initial model of lysine biosynthesis. They then "trained" the model to match high-throughput experimental results. Training an initial qualitative model to produce a robust refined quantitative model that is more relevant is done through the cyclic process: simulate, test, and refine the model. Imoto and coworkers $^{98}$ realized that constructing networks from time-series microarray data alone is not sufficient to build accurate models that can be used to make quality predictions on cellular behavior. They combined protein-protein, protein-DNA binding sites, and literature data with microarray data to construct a more realistic network of a yeast gene regulation network by searching for computational models that can fit both the highthroughput experiments and the data gathered from literature. Implementing gradient-descent types of searches for the purpose of fitting simulated networks inferred from a combination of different studies, they identified network topologies that can explain best the results from the microarray time series data. Integration of literature data with high-throughput experiments is the most promising method toward high-quality computational models because it capitalizes on the greatest amount of experimental evidence. ${ }^{94}$

\section{MOUSE MODELS}

Predictions of pathway perturbations produced by computational approaches will be first tested using mouse models. This requires the construction of appropriate segmental trisomies. Of the $\sim 170$ chromosome 21 protein-coding genes, $\sim 110$ are conserved in the telomeric segment of mouse chromosome 16 , and $\sim 20$ are found in a centromere proximal segment of mouse chromosome 17 , whereas $\sim 40$ are located in an internal segment of mouse chromosome $10 .{ }^{12}$ Creating a "perfect" mouse model of DS is thus a formidable challenge. Currently, the most complete model is the Ts65Dn, which is trisomic for a telomeric segment of mouse chromosome 16 containing 94 orthologs of chromosome 21 proteins. The Ts65Dn displays many interesting and critical features relevant to DS, including behavioral deficits, abnormalities of dendritic spines, and loss of cholinergic neuronal markers (reviewed in $^{499-100}$ ). However, because it is not trisomic for 76 of the known protein coding genes, it cannot be assumed to recapitulate the complete phenotype DS. Indeed, it cannot recapitulate perturbations known and predicted to occur in many of the networks of interacting proteins listed in Table 1 and shown in Figures 1 and 2 because it lacks an 
extra copy of one or more components. Thus, more complete models are required. One such model that was reported recently ${ }^{101}$ is a mouse that carries a human chromosome 21. Drawbacks to this model, in addition to being a heterologous system, are that the mice are mosaics (not all cells in an individual contain the extra chromosome, and the proportions of trisomic cells vary among mice). Additionally, the chromosome 21 carries an internal deletion of some possibly important genes. Two additional mouse chromosome 16 segmental trisomies are available. The Ts1Cje and the Ts1Rrh are trisomic for $\sim 70$ and $\sim 30$ orthologs of chromosome 21 proteins, respectively. Subsets of those genes are also in the Ts65Dn model. ${ }^{102,103}$

Thus, comparisons between these models of behavioral, cellular, and molecular phenotypes may provide information on the contributions of sets of genes. However, systematic comprehensive understanding requires more deliberate construction of DS models, ones that consider the predictions of pathway contributions by specific chromosome 21 genes and sets of genes. These should include adding to the Ts65Dn, Ts1Cje, or Ts1Rrh specific genes and sets of genes (e.g., as BAC transgenics overexpressing one or more genes), to provide trisomy for complete pathway components, and subtracting from the Ts65Dn or other segmental trisomies, individual genes (e.g., single-gene knockouts). This would correlate phenotypes with subsets of genes and would verify their predicted contributions to specific pathways. Pathway perturbations can be further tested by exposing mice to tests of learning and behavior that require candidate pathways and by treating mice with drugs that perturb or correct perturbations in candidate pathways. Generation of appropriate mouse models is technically feasible but time consuming. Assaying pathway perturbations at the protein level and, importantly, at the level of post-translational modifications is challenging because it requires sensitivity (perturbations observed in the Ts65Dn so far are modest, $<50 \%{ }^{27}$ ). In addition, large-scale, highthroughput proteomics approaches would be required to assay multiple brain regions from multiple mouse models, at different ages, with and without behavioral or pharmacological treatments.

\section{CONCLUSION}

Data from cell- and tissue-based assays applied to cells and tissues from mouse models of DS or cells from humans with DS can be combined with networks, such as those shown in Figures 1 and 2 to build the next generation of computational models with predictive capabilities (FIG. 3). Searching for a network topology that can reproduce the experimental observations is challenging but has great potential to speed our understanding of the phenotypic differences observed in cellular dynamics in DS. There are 16,857 articles returned from a PubMed

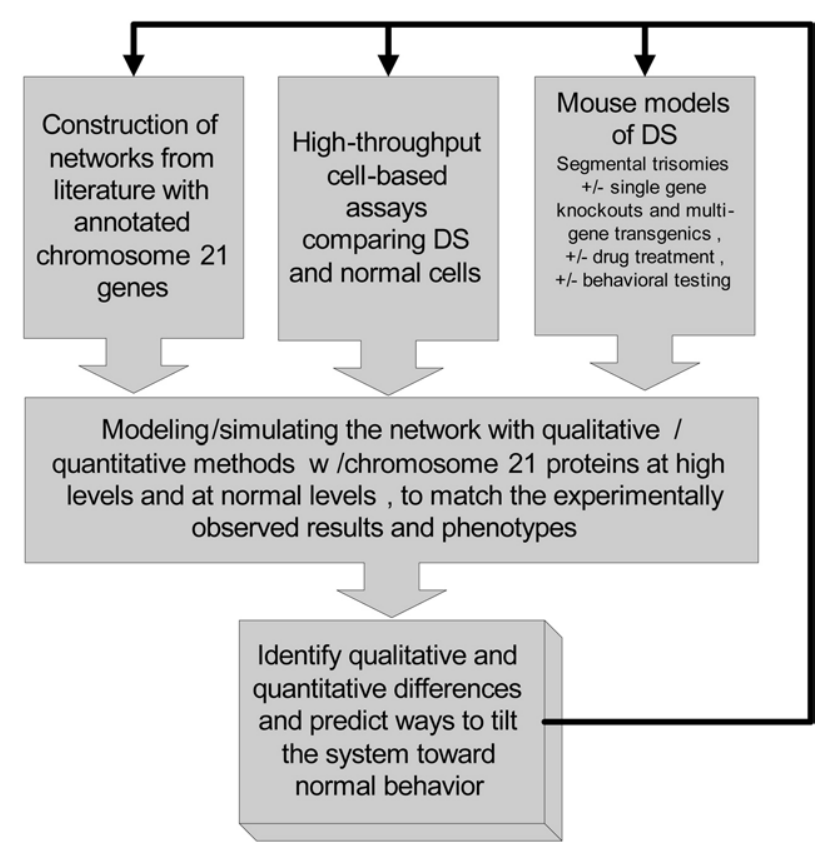

FIG. 3. Plan for approaching Down syndrome research using systems biology methodologies.

search using the term "Down syndrome" but none when the term "systems biology" is added. The emerging field of systems biology could provide an accelerator toward eventual therapeutics for DS. It is not expected that drugs would abolish all DS phenotypic features but they can be expected to improve cognitive function and help to prolong life span. Additionally, understanding early onset AD-like neuropathology and cognitive decline in DS from a systems perspective can advance our understanding of AD in general. Systematic approaches have been already applied to understand systematic properties of AD (for example see Ginsberg and coworkers ${ }^{104}$ in this issue). The application of high-throughput methods to tackle specific cellular diseases and to identify biomarkers to classify different disease states in individuals is not just a trend but a paradigm shift. Other neuronal disorders such as Parkinson's disease (reviewed by Miller and Federoff $^{105}$ in this issue) or drug addiction (reviewed by $\mathrm{Uhl}^{106}$ in this issue) are already benefiting from such emerging technologies. Modeling using graph theory and other newly developed computational tools, combining data gathered through literature mining with datasets produced through high-throughput experiments, are surely required for, and will be successful in, comprehensive understanding of complex neuronal disorders such as DS.

Acknowledgments: A. Ma'ayan was supported by the National Institutes of Health (NIH) Training Grant GM-62754. Research in the Iyengar Laboratory is supported by Grants GM-072853 and GM-54508 from NIH and an advanced center grant from NYSTAR. Research in the Gardiner Laboratory is 
supported by Grants HD047671 and HD49460 from NIH and by the Foundation Jerome Lejeune.

\section{REFERENCES}

1. Hassold TJ, Jacobs PA. Trisomy in man. Annu Rev Genet 18:6997, 1984.

2. Epstein CJ. Down's syndrome (trisomy 21). In: Metabolic and molecular basis of inherited disease (Scriver, CA, Beaudet AL, Sly WS, Valle D, eds), pp 749-794. New York: McGraw Hill, 1995.

3. Nadel L. Down's syndrome: a genetic disorder in biobehavioral perspective. Genes Brain Behav 2:156-166, 2003.

4. Benavides-Piccione R, Ballesteros-Yanez I, de Lagran MM, Elston G, Estivill X, Fillat C, et al. On dendrites in Down's syndrome and DS murine models: a spiny way to learn. Prog $\mathrm{Neu}$ robiol 74:111-126, 2004.

5. Chapman RS, Hesketh LJ. Behavioral phenotype of individuals with Down's syndrome. Ment Retard Dev Disabil Res Rev 6:8495, 2000.

6. Tolmie JL. Down's syndrome and other autosomal trisomies. In: Principles and practices of medical genetics (Rimoin D, O'Connor JM, Pyeritz RE, Emergy AEH, eds), Ed 3, Ch 47, pp 925-971. Scotland: WB Saunders, Livingstone, 1997.

7. Law G, Byrne A, Buckley S. Language and memory development in children with Down's syndrome at mainstream and special schools: a comparison. Educ Psychol 20:447-445, 7.

8. Brock J, Jarrold C. Serial order reconstruction in Down's syndrome: evidence for a selective deficit in verbal short-term memory. J Child Psychol Psychiatry 46:304-316, 2005.

9. Pennington BF, Moon J, Edgin J, Stedron J, Nadel L. The neuropsychology of Down's syndrome: evidence for hippocampal dysfunction. Child Dev 74:75-93, 2003.

10. Lott IT, Head E. Alzheimer disease and Down's syndrome: factors in pathogenesis. Neurobiol Aging 26:383-389, 2005.

11. Roizen NJ, Patterson D. Down's syndrome. Lancet 361:12811289, 2003.

12. Gardiner K, Fortna A, Bechtel L, Davisson MT. Mouse models of Down's syndrome: how useful can they be? Comparison of the gene content of human chromosome 21 with orthologous mouse genomic regions. Gene 318:137-147, 2003.

13. Delabar JM, Creau N, Sinet PM, Ritter O, Antonarakis SE, Burmeister M, et al. Report of the Fourth International Workshop on Human Chromosome 21. Genomics 18:735-745, 1993.

14. Rahmani Z, Blouin JL, Creau-Goldberg N, Watkins PC, Mattei $\mathrm{JF}$, Poissonnier M, et al. Down's syndrome critical region around D21S55 on proximal 21q22.3. Am J Med Genet Suppl 7:98-103, 1990.

15. Korenberg JR, Kawashima H, Pulst SM, Ikeuchi T, Ogasawara N, Yamamoto K, et al. Molecular definition of a region of chromosome 21 that causes features of the Down's syndrome phenotype. Am J Hum Genet 47:236-246, 1990.

16. Korenberg JR, Chen XN, Schipper R, Sun Z, Gonsky R, Gerwehr $\mathrm{S}$, et al. Down's syndrome phenotypes: the consequences of chromosomal imbalance. Proc Natl Acad Sci USA 91:4997-5001, 1994.

17. Dauphinot L, Lyle R, Rivals I, Dang MT, Moldrich RX, Golfier $\mathrm{G}$, et al. The cerebellar transcriptome during postnatal development of the Ts1Cje mouse, a segmental trisomy model for Down's syndrome. Hum Mol Genet 14:373-384, 2005.

18. Kahlem P, Sultan M, Herwig R, Steinfath M, Balzereit D, Eppens $\mathrm{B}$, et al. Transcript level alterations reflect gene dosage effects across multiple tissues in a mouse model of Down's syndrome. Genome Res 14:1258-1267, 2004.

19. Lyle R, Gehrig C, Neergaard-Henrichsen C, Deutsch S, Antonarakis SE. Gene expression from the aneuploid chromosome in a trisomy mouse model of Down's syndrome. Genome Res 14:1268-1274, 2004.

20. Amano K, Sago H, Uchikawa C, Suzuki T, Kotliarova SE, Nukina N, et al. Dosage-dependent over-expression of genes in the trisomic region of Ts1Cje mouse model for Down's syndrome. Hum Mol Genet 13:1333-1340, 2004.
21. Mao R, Zielke CL, Zielke HR, Pevsner J. Global up-regulation of chromosome 21 gene expression in the developing Down's syndrome brain. Genomics 81:457-467, 2003.

22. Gardiner K. Gene-dosage effects in Down's syndrome and trisomic mouse models. Genome Biol 5:244, 2004.

23. Deutsch S, Lyle R, Dermitzakis ET, Attar H, Subrahmanyan L, Gehrig C, et al. Gene expression variation and expression quantitative trait mapping of human chromosome 21 genes. Hum Mol Genet 14:3741-3749, 2005.

24. O'Leary DA, Pritchard MA, Xu D, Kola I, Hertzog PJ, Ristevski S. Tissue-specific overexpression of the HSA21 gene GABPalpha: implications for DS. Biochim Biophys Acta 1739:81-87, 2004.

25. Gardiner K, Costa AC. The proteins of human chromosome 21. Am J Med Genet (in press).

26. Nikolaienko O, Nguyen C, Crinc LS, Cios KJ, Gardiner K. Human chromosome 21/Down's syndrome gene function and pathway database. Gene 364:90-98, 2005.

27. Gardiner K, Davisson MT, Crnic LS. Building protein interaction maps for Down's syndrome. Brief Funct Genomic Proteomic 3:142-156, 2004.

28. Rothermel BA, Vega RB, Williams RS. The role of modulatory calcineurin-interacting proteins in calcineurin signaling. Trends Cardiovasc Med 13:15-21, 2003.

29. DasGupta R, Kaykas A, Moon RT, Perrimon N. Functional genomic analysis of the Wnt-wingless signaling pathway. Science 308:826-833, 2005 .

30. Ozawa S, Kamiya H, Tsuzuki K. Glutamate receptors in the mammalian central nervous system. Prog Neurobiol 54:581-618, 1998.

31. Cooper JD, Salehi A, Delcroix JD, Howe CL, Belichenko PV, Chua-Couzens J, et al. Failed retrograde transport of NGF in a mouse model of Down's syndrome: reversal of cholinergic neurodegenerative phenotypes following NGF infusion. Proc Natl Acad Sci USA 98:10439-10444, 2001.

32. Roper RJ, Baxter LL, Saran NG, Klinedinst DK, Beachy PA, Reeves RH. Defective cerebellar response to mitogenic Hedgehog signaling in Down's syndrome mice. Proc Natl Acad Sci USA 03:1452-1456, 2006.

33. Granholm AC, Ford KA, Hyde LA, Bimonte HA, Hunter CL, Nelson M, et al. Estrogen restores cognition and cholinergic phenotype in an animal model of Down's syndrome. Physiol Behav 77:371-385, 2002.

34. Clark S, Schwalbe J, Stasko MR, Yarowsky PJ, Costa ACS. Fluoxetine rescues deficient neurogenesis in hippocampus of the mouse model for Down's syndrome Ts65Dn. Exp Neurol (in press).

35. Li W, Kurata H. A grid layout algorithm for automatic drawing of biochemical networks. Bioinformatics 21:2036-2042, 2003.

36. Gardner TS, di Bernardo D, Lorenz D, Collins JJ. Inferring genetic networks and identifying compound mode of action via expression profiling. Science 301:102-105, 2003.

37. Ito T, Chiba T, Ozawa R, Yoshida M, Hattori M, Sakaki Y. A comprehensive two-hybrid analysis to explore the yeast protein interactome. Proc Natl Acad Sci USA 98:4569-4574, 2001.

38. Giot L, Bader JS, Brouwer C, Chaudhuri A, Kuang B, Li Y, et al. A protein interaction map of Drosophila melanogaster. Science 302:1727-1736, 2003.

39. Li S, Armstrong CM, Bertin N, Ge H, Milstein S, Boxem M, et al. A map of the interactome network of the metazoan C. elegans. Science 303:540-543, 2004.

40. Rual JF, Venkatesan K, Hao T, Hirozane-Kishikawa T, Dricot A, $\mathrm{Li} \mathrm{N}$, et al. Towards a proteome-scale map of the human proteinprotein interaction network. Nature 437:1173-1178, 2005.

41. Stelzl U, Worm U, Lalowski M, Haenig C, Brembeck FH, Goehler $\mathrm{H}$, et al. A human protein-protein interaction network: a resource for annotating the proteome. Cell 122:957-968, 2005.

42. Nuhse TS, Stensballe A, Jensen ON, Peck SC. Large-scale analysis of in vivo phosphorylated membrane proteins by immobilized metal ion affinity chromatography and mass spectrometry. Mol Cell Proteomics 2:1234-1243, 2003.

43. Irish JM, Hovland R, Krutzik PO, Perez OD, Bruserud O, Gjert- 
sen BT, et al. Single cell profiling of potentiated phospho-protein networks in cancer cells. Cell 118:217-228, 2004.

44. Ptacek J, Devgan G, Michaud G, Zhu H, Zhu X, Fasolo J, et al. Global analysis of protein phosphorylation in yeast. Nature 438: 679-684, 2005.

45. Jones RB, Gordus A, Krall JA, MacBeath G. A quantitative protein interaction network for the ErbB receptors using protein microarrays. Nature 439:168-174, 2006.

46. Friedman C, Kra P, Yu H, Krauthammer M, Rzhetsky A. GENIES: a natural-language processing system for the extraction of molecular pathways from journal articles. Bioinformatics 17: S74-S82, 2002.

47. Marcotte EM, Xenarios I, Eisenberg D. Mining literature for protein-protein interactions. Bioinformatics 17:359-363, 2001.

48. Nikitin A, Egorov S, Daraselia N, Mazo I. Pathway studio: the analysis and navigation of molecular networks. Bioinformatics 19:2155-2157, 2004.

49. Daraselia N, Yuryev A, Egorov S, Novichkova S, Nikitin A, Mazo I. Extracting human protein interactions from MEDLINE using a full-sentence parser. Bioinformatics 20:604-611, 2004.

50. Mishra GR, Suresh M, Kumaran K, Kannabiran N, Suresh S, Bala P, et al. Human protein reference database: 2006 update. Nucleic Acids Res 34:D411-D414, 2006.

51. Gough NR. Science's signal transduction knowledge environment: the connections maps database. Ann NY Acad Sci 971:585587, 2002.

52. Ma'ayan A, Jenkins SL, Neves S, Hasseldine A, Grace E, DubinThaler B, et al. Formation of regulatory patterns during signal propagation in a mammalian cellular network. Science 309:1078, 2005.

53. von Mering C, Krause R, Snel B, Cornell M, Oliver SG, Fields S, et al. Comparative assessment of large-scale data sets of proteinprotein interactions. Nature 417:399-403, 2002.

54. Hucka M. The systems biology markup language (SBML): a medium for representation and exchange of biochemical network models. Bioinformatics 19:524-531, 2003.

55. Sivakumaran S, Hariharaputran S, Mishra J, Bhalla US. The Database of Quantitative Cellular Signaling: management and analysis of chemical kinetic models of signaling networks. Bioinformatics 19:408-415, 2003.

56. Bader GD, Betel D, Hogue CW. BIND: the Biomolecular Interaction Network Database. Nucleic Acids Res 31:248-250, 2003.

57. Zanzoni A, Montecchi-Palazzi L, Quondam M, Ausiello G, Helmer-Citterich M, Cesareni G. MINT: a Molecular INTeraction database. FEBS Lett 513:135-140, 2002.

58. Hermjakob H, Montecchi-Palazzi L, Lewington C, Mudali S, Kerrien S, Orchard S, et al. IntAct: an open source molecular interaction database. Nucleic Acids Res 32:D452-D455, 2004.

59. Xenarios I, Rice DW, Salwinski L, Baron MK, Marcotte EM, Eisenberg D. DIP: The Database of Interacting Proteins: 2001 update. Nucleic Acids Res 29:239-241, 2001.

60. Salwinski L, Miller CS, Smith AJ, Pettit FK, Bowie JU, Eisenberg D. The Database of Interacting Proteins: 2004 update. $\mathrm{Nu}$ cleic Acids Res 32:D449-D451, 2004.

61. Kanehisa M, Goto S, Kawashima S, Nakaya A The KEGG databases at GenomeNet. Nucleic Acids Res 30:42-46, 2002.

62. Choudhary J, Grant SG. Proteomics in postgenomic neuroscience: the end of the beginning. Nat Neurosci 7:440-445, 2004.

63. Hermjakob H, Montecchi-Palazzi L, Bader G, Wojcik J, Salwinski L, Ceol A, et al. The HUPO PSI's molecular interaction format-a community standard for the representation of protein interaction data. Nat Biotechnol 22:177-183, 2004.

64. Bader GD, Cary MP, Sander C. Pathguide: a pathway resource list. Nucleic Acids Res 34:D504-D506, 2006.

65. Gerdes AM, Horder M, Bonnevie-Nielsen V. Increased IFN- $\alpha$ induced sensitivity but reduced reactivity of $2^{\prime}, 5^{\prime}$-oligoadenylate synthetase $(2,5 \mathrm{AS})$ in trisomy 21 blood lymphocytes. Clin Exp Immunol 93:93-96, 1993.

66. Albert R. Scale-free networks in cell biology. J Cell Sci 118: 4947-4957, 2005

67. Watts DJ, Strogatz SH. Collective dynamics of 'small-world' networks. Nature 393:440-442, 1998.
68. Jeong H, Mason SP, Barabasi AL, Oltvai ZN. Lethality and centrality in protein networks. Nature 411:41-42, 2001.

69. Milo R, Shen-Orr S, Itzkovitz S, Kashtan N, Chklovskii D, Alon U. Network motifs: simple building blocks of complex networks. Science 298:824-827, 2002.

70. Han JD, Bertin N, Hao T, Goldberg DS, Berriz GF, Zhang LV, et al. Evidence for dynamically organized modularity in the yeast protein-protein interaction network. Nature 430:88-93, 2004.

71. Borneman AR, Leigh-Bell JA, Yu H, Bertone P, Gerstein M, Snyder M. Target hub proteins serve as master regulators of development in yeast. J Cataract Refract Surg 31:2051-2054, 2005.

72. Ma'ayan A, Blitzer RD, Iyengar R. Toward predictive models of mammalian cells. Апnи Rev Biophys Biomol Struct 34:319-349, 2005.

73. Bornholdt S. Systems biology: less is more in modeling large genetic networks. Science 310:449-451, 2005.

74. Tyson JJ, Chen K, Novak B. Network dynamics and cell physiology. Nat Rev Mol Cell Biol 2:908-916, 2002.

75. Kuipers B. Qualitative reasoning: modeling and simulation with incomplete knowledge. Cambridge, MA: MIT Press, 1994.

76. King RD, Garrett SM, Coghill GM. On the use of qualitative reasoning to simulate and identify metabolic pathways. Bioinformatics 21:2017-2026, 2005.

77. Albert R, Othmer HG. The topology of the regulatory interactions predicts the expression pattern of the segment polarity genes in Drosophila melanogaster. J Theor Biol 223:1-18, 2003.

78. Grefenstette J, Kim S, Kauffman S. An analysis of the class of gene regulatory functions implied by a biochemical model. Biosystems (10.1016/j.biosystems. 2005.09.009), 26 December 2005.

79. Oliveira JS, Jones-Oliveira JB, Dixon DA, Bailey CG, Gull DW. Hyperdigraph-theoretic analysis of the EGFR signaling network: initial steps leading to GTP:Ras complex formation. J Comput Biol 11:812-842, 2005.

80. Bhalla US, Iyengar R. Emergent properties of networks of biological signaling pathways. Science 283:381-387, 1999.

81. Goss PJ, Peccoud J. Quantitative modeling of stochastic systems in molecular biology by using stochastic Petri nets. Proc Natl Acad Sci USA 95:6750-6755, 1998.

82. Holland JH. Exploring the evolution of complexity in signaling networks. Complexity 7:34, 2002.

83. Yang D, Zakharkin SO, Page GP, Brand JP, Edwards JW, Bartolucci AA, et al. Applications of Bayesian statistical methods in microarray data analysis. Am J Pharmacogenomics 4:53-62, 2004.

84. Friedman N, Linial M, Nachman I, Pe'er D. Using Bayesian networks to analyze expression data. J Comput Biol 7:601-620, 2001.

85. Sachs K, Gifford D, Jaakkola T, Sorger P, Lauffenburger DA. Bayesian network approach to cell signaling pathway modeling. SCi STKE 2002:PE38, 2002.

86. Woolf PJ, Prudhomme W, Daheron L, Daley GQ, Lauffenburger DA. Bayesian analysis of signaling networks governing embryonic stem cell fate decisions. Bioinformatics 21:741-753, 2005.

87. Prudhomme W, Daley GQ, Zandstra P, Lauffenburger DA. Multivariate proteomic analysis of murine embryonic stem cell selfrenewal versus differentiation signaling. Proc Natl Acad Sci USA 101:2900-2905, 2004.

88. Sachs K, Perez O, Pe'er D, Lauffenburger DA, Nolan GP. Causal protein-signaling networks derived from multiparameter singlecell data. Science 308:523-529, 2005.

89. Dean T, Kanazawa K. A model for reasoning about persistence and causation. Comput Intell 5:142-150, 1989.

90. Zou M, Conzen SD. A new dynamic Bayesian network (DBN) approach for identifying gene regulatory networks from time course microarray data. Bioinformatics 21:71-79, 2005.

91. Murphy K, Mian S. Modeling gene expression data using dynamic Bayesian networks. Technical report, Computer Science Division, University of California, Berkeley, 1999.

92. Segal E, Pe'er D, Regev A, Koller D, Friedman N. Learning Module Networks. J Machine Learn Res 6:557-588, 2005.

93. Janes KA, Albeck JG, Gaudet S, Sorger PK, Lauffenburger DA, Yaffe MB. A systems model of signaling identifies a molecular 
basis set for cytokine-induced apoptosis. Science 310: 1646-1653, 2005.

94. Ma'ayan A, Iyengar R. From components to regulatory motifs in signalling networks. Brief Funct Genomic Proteomic 5:57-61, 2006.

95. Kurata H, Masaki K, Sumida Y, Iwasaki R. CADLIVE dynamic simulator: direct link of biochemical networks to dynamic models. Genome Res 15:590-600, 2005.

96. D'haeseleer P, Liang S, Somogyi R. Genetic network inference: from co-expression clustering to reverse engineering. Bioinformatics 16:707-726, 2000.

97. Gat-Viks I, Tanay A, Shamir R. Modeling and analysis of heterogeneous regulation in biological networks. J Comput Biol 11: 1034-1049, 2005.

98. Imoto S, Iguchi T, Goto T, Tashiro K, Kuhara S, Miyano S. Combining microarrays and biological knowledge for estimating gene networks via bayesian networks. J Bioinform Comput Biol 2:77-98, 2003.

99. Costa AC, Walsh K, Davisson MT. Motor dysfunction in a mouse model for Down's syndrome. Physiol Behav 68:211-220, 1999.
100. Crnic LS, Pennington BF. Down's syndrome: neuropsychology and animal models. Progr Infancy Res 1:69-111, 2000.

101. O'Doherty A, Ruf S, Mulligan C, Hildreth V, Errington ML, Cooke S, et al. An aneuploid mouse strain carrying human chromosome 21 with Down's syndrome phenotypes. Science 309: 2033-2037, 2005.

102. Sago H, Carlson EJ, Smith DJ, Kilbridge J, Rubin EM, Mobley WC, et al. Ts1Cje, a partial trisomy 16 mouse model for Down's syndrome, exhibits learning and behavioral abnormalities. Proc Natl Acad Sci USA 95:6256-6261, 1998.

103. Olson LE, Richtsmeier JT, Leszl J, Reeves RH. A chromosome 21 critical region does not cause specific Down's syndrome phenotypes. Science 306:687-690, 2004.

104. Ginsberg SD, Che S, Counts SE, Mufson, EJ. Single cell gene expression profiling in Alzheimer's disease. NeuroRx, 3:302-317, 2006.

105. Miller RM, Federoff HJ. Microarrays in Parkinson's disease: a systematic approach. NeuroRx 3:318-325, 2006.

106. Uhl GR. Molecular genetics of addiction vulnerability. NeuroRx, 3:295-301, 2006 